\title{
Frustrated quantum Heisenberg ferrimagnetic chains
}

\author{
N. B. Ivanov \\ Institute for Solid State Physics, Bulgarian Academy of Sciences, \\ Tzarigradsko chaussee-72, 1784 Sofia, Bulgaria \\ J. Richter \\ Institut für Theoretische Physik, Universität Magdeburg, \\ P.O.Box 4120, D-39016 Magdeburg, Germany \\ U. Schollwöck \\ Sektion Physik, Ludwig-Maximilians-Universität München, \\ Theresienstrasse 37/III, 80333 Munich, Germany
}

\begin{abstract}
We study the ground-state properties of weakly frustrated Heisenberg ferrimagnetic chains with nearest and next-nearest neighbor antiferromagnetic exchange interactions and two types of alternating sublattice spins $S_{1}>S_{2}$, using $1 / S$ spin-wave expansions, density-matrix renormalization group, and exact-diagonalization techniques. It is argued that the zero-point spin fluctuations completely destroy the classical commensurate-incommensurate continuous transition. Instead, the long-range ferrimagnetic state disappears through a discontinuous transition to a singlet state at a larger value of the frustration parameter. In the ferrimagnetic phase we find a disorder point marking the
\end{abstract}


onset of incommensurate real-space short-range spin-spin correlations.

PACS: 75.50.Ee, 75.10.Jm, 75.30.Kz, 75.10.-b

Typeset using REVTEX 


\section{INTRODUCTION}

Over the last years a great deal of interest has been concentrated on the physics of quantum Heisenberg spin chains with competing exchange couplings both for half-integer and integer site spins (see, e.g., Refs. [1] 3] and the references therein). In general, frustration reduces the antiferromagnetic correlations and in some cases may produce various exotic quantum ground states such as the dimerized state, the spin-nematic state, or some sort of spin-liquid states. At the same time, little is known about the role of frustration in the so-called mixed spin chains constructed from two or more kinds of site spins regularly distributed on the lattice. Recently, a number of studies concerning the physics of unfrustrated mixed Heisenberg chains with two kinds of spins have been published 4 田. Depending on the periodic array, the mixed models can form massive or massless ground states and display a rich variety of new features [6,77. Meanwhile, various mixed one-dimensional quantum spin systems have already been synthesized in the last decade [8].

In this report we address one-dimensional spin chains containing two different alternating site spins $S_{1}>S_{2}$ per unitary cell and interacting via competing antiferromagnetic neighbor and next-neighbor couplings, $J_{1}>0$ and $J_{2}>0$, Fig.1. The Hamiltonian of the system reads:

$$
H=J_{1} \sum_{n, \delta} \mathbf{S}_{\mathbf{1}_{n}} \mathbf{S}_{\mathbf{2}_{n+\delta}}+J_{2} \sum_{n}\left(\mathbf{S}_{\mathbf{1}_{n}} \mathbf{S}_{\mathbf{1}_{n+1}}+\mathbf{S}_{\mathbf{2}_{n}} \mathbf{S}_{\mathbf{2} n+1}\right)
$$

where the integers $n$ number $N$ unitary cells and the vector $\delta= \pm 1 / 2$ connects the $S_{1}$ spins with the nearest-neighbor $S_{2}$ spins. The size of the elementary cell is unity. In what follows we frequently use the notations $w \equiv S_{1} / S_{2}, S \equiv S_{2}, J \equiv J_{2} / J_{1}, J_{1} \equiv 1$. Theoretical models of quantum ferrimagnetic systems with competing interactions have already been discussed in the literature [9]. However, these models consider complicated multiple spinspin interactions which are far from the real experiment. On the other hand, little is known about the simple frustrated Heisenberg models containing only two-spin couplings.

In the classical limit, the ground state of Eq.(11) can be described by the ansatz: 


$$
\mathbf{S}_{\mathbf{i} n}=S_{i}[\hat{\mathbf{u}} \cos (Q n)+\hat{\mathbf{v}} \sin (Q n)], \quad i=1,2,
$$

where $\hat{\mathbf{u}} \perp \hat{\mathbf{v}}$ are unit vectors in the spin space. The classical commensurate ferrimagnetic state with a pitch angle between the nearest spins $\theta \equiv Q / 2=\pi$ is stable up to the phase transition point

$$
J_{C}=\frac{w}{2\left(w^{2}+1\right)} .
$$

For larger values of frustration, $J>J_{C}$, the stable state is a spiral state with an ordering wave vector given by

$$
\cos \frac{Q}{2}=-\frac{w}{2 J\left(w^{2}+1\right)} .
$$

In the limit $J=\infty, \theta=\pi / 2$ and the system is composed of two decoupled antiferromagnetic chains with site spins $S_{1}$ and $S_{2}$. The long-range Néel order is excluded in this limit, but one might expect the classical result to give a guide to possible finite-range order. In the extreme antiferromagnetic case, $w=1$, Eq. (44) reduces to the well-known result for homogenous frustated antiferromagnetic chains, and $J_{C}$ reaches its upper bound $1 / 4$. Recent studies of

the $J=0$ system confirm the expectation that the classical ferrimagnetic state survives the quantum spin fluctuations [ [. longitudinal ferromagnetic and antiferromagnetic long-range orders [5]. Thus, a special feature of the discussed model, Eq. (11), is the existence of an order-disorder quantum phase transition. The character of the latter transition should crucially depend on the values of quantum site spins $S_{1}$ and $S_{2}$.

\section{SPIN-WAVE ANALYSIS}

In this report we consider relatively small values of the frustration parameter $J$. In the unfrustrated case, the linear spin-wave theory (LSWT) has already been successfully applied in a series of recent works 四. The second-order $1 / S$ series for a number of quantities were shown [10] to reproduce with a high precision the density matrix renormalization group 
(DMRG) results. Thus, we can expect that at least in the weakly frustrated region the spin-wave theory (SWT) gives a realistic picture of the low-energy physics of the system. However, its application to other frustrated spin systems reveals that in many cases the linear theory gives better qualitative results [11]. In this respect, we observe similar behavior in the present system, so that the use of spin-wave expansions requires some care.

In the ferrimagnetic region LSWT predicts the existence of two types of elementary excitations:

$$
E_{k}^{(a, b)}=2 S\left(\frac{a_{k}+b_{k}}{2} \epsilon_{k} \pm \frac{\left(a_{k}-b_{k}\right)}{2}\right)
$$

where $a_{k}=1-w(1-\cos k), b_{k}=w-J(1-\cos k), \epsilon_{k}=\sqrt{1-\eta_{k}^{2}}$, and $\eta_{k}=2 \sqrt{w} \cos (k / 2) /\left(a_{k}+\right.$ $b_{k}$ ). The $E_{k}^{(a)}$ excitations are gapless, $E_{k}^{(a)} \sim k^{2}$ for small $k$. They describe ferromagnetic magnons in the sector with a total spin $\left(S_{1}-S_{2}\right) N-1$. The $E_{k}^{(b)}$ excitations are gapful, $E_{k}^{(b)}=\Delta+O\left(k^{2}\right)$, and belong to the sector $\left(S_{1}-S_{2}\right) N+1$ (antiferromagnetic magnons). The existence of gapless excitations at $k=0$ reflects the continuous symmetry of the Hamiltonian under a global rotation of spins. On the other hand, for $w>1$ the antiferromagnetic excitations acquire a gap due to the violated sublattice symmetry. In the weakly frustrated region the structure of low-lying energy levels can also be predicted from the Lieb-Mattis theorem [12]: For every finite $N$, the energy levels order according to the rule:

$$
\begin{gathered}
E\left(S_{t}+1\right)>E\left(S_{t}\right), \quad, S_{t} \geq S_{g}, \\
E\left(S_{t}\right)>E\left(S_{g}\right), \quad, S_{t}<S_{g}
\end{gathered}
$$

where $S_{t}$ is the total spin of the state and $S_{g}=\left(S_{1}-S_{2}\right) N$ is the spin of the ground state. Strictly speaking, the Lieb-Mattis theorem does not work in the frustrated system, but due to the continuity principle we can expect that the order of states survives up to some finite $J$ [13]. The above picture is also confirmed in our exact-diagonalization studies of small clusters. On the other hand, the strong frustration might cause a substantial disarrangement of levels, as is shown numerically below. 
Exactly at the classical transition point $J=J_{C}$, the quadratic term in the small $k$ expansion of $E_{k}^{(a)}$ vanishes, so that the spectrum reads: $E_{k}^{(a)}=c k^{4}+O\left(k^{6}\right), \quad c>0, J=J_{C}$. For $J>J_{C}$, the classical ground state is the spiral state described above, Eq.(四). Now LSWT predicts zero modes at $k=0$ and at $k= \pm(2 \pi-Q)$. However, the classically broken $\mathrm{SO}(3)$ symmetry in the spiral state is generally expected to be restored by quantum fluctuations in one-dimensional systems [14], so that a crucial role of boson-boson interactions should be expected near and beyond the classical transition point.

It is instructive to trace back the change in the gap function $\Delta(J)$. The first two terms in the $1 / S$ series are:

$$
\Delta=(w-1)\left(2 S-\frac{2 g_{1}}{\sqrt{w}}\right)+O\left(\frac{1}{S}\right), \quad g_{1}=-\frac{1}{2 N} \sum_{k} \frac{\eta_{k}}{\epsilon_{k}} \cos \frac{k}{2} .
$$

In Fig. 2 we show the dispersion functions, Eq. (5), in the case $\left(S_{1}, S_{2}\right)=(1,1 / 2)$ for different frustration parameters $J$. Near the transition point the ferromagnetic mode is strongly flatted, whereas the changes in $E_{k}^{(b)}$ are modest. In addition, Fig. 3 displays a smooth increase of the gap $\Delta$ up to the classical transition point $J_{C}$, which means that the antiferromagnetic excitations do not play any important role in the mechanism of the transition.

In Figs. 4 and 5 second-order SWT results for the ground state energy and the sublattice magnetization are compared to those of the DMRG and the exact diagonalization (ED) numerical methods. SWT values for the energy are close to the numerical ones in a large region of the ferrimagnetic phase. As to the magnetization, SWT is effective only in a small vicinity of the unfrustrated model. Such a collapse of spin-wave series has already been indicated in other frustrated spin models [15]. We are faced with an example where the linear theory gives a better qualitative description.

Let us now address the spin-spin correlations. The latter will be analysed quantitatively in the framework of the DMRG method below. Here the purpose is to demonstrate that LSWT qualitatively captures the asymptotic behavior of the finite-range spin-spin correlations. As an example, we consider the spin-spin correlator $K_{12}^{\perp}(r)=\left\langle\mathbf{S}_{\mathbf{1}_{n}}^{+} \mathbf{S}_{\mathbf{2}}^{-}{ }_{n+r}+\right.$ 
$\left.\mathbf{S}_{\mathbf{1}_{n}}^{-} \mathbf{S}_{\mathbf{2}}^{+}{ }_{n+r}\right\rangle / 2$. In a linear spin-wave approximation we have:

$$
K_{12}^{\perp}(r)=-\frac{2 S \sqrt{w}}{N} \sum_{k} \frac{\eta_{k}}{\epsilon_{k}} \cos (k r) .
$$

The asymptotic behavior of $K_{12}^{\perp}(r)$ for long distances $r$ depends on the analytic structure of the dominator in Eq. (9). It is easy to check that in the region $0<J<J_{D}$,

$$
J_{D}=\frac{1}{2(w+1)},
$$

the poles in Eq. (9) are pure imaginary. Using an appropriate standard integral, on obtains the following two-dimensional (2D) Ornstein-Zernike type asyptotic expression:

$$
K_{12}^{\perp}(r) \sim \frac{e^{-\frac{r}{\xi}}}{\sqrt{r}}, \quad r \rightarrow \infty, \quad J<J_{D} .
$$

This can be generically expected for a one-dimensional quantum problem from fieldtheoretical arguments. $\xi$ is the correlation length of the short-range transverse spin fluctuations. In the limit $J=0$, LSWT predicts $\xi=1 / \ln w$, as it was also obtained by Pati et al. 四. The LSWT function $\xi(J), 0 \leq J \leq J_{D}$, compared to the DMRG results, is shown in Fig. 6. A well-pronounced effect of fructration is the reduction of the correlation length. Qualitatively, LSWT reproduces the latter tendency. The special point $J_{D}$, known as a disorder point of the first kind in classical thermodynamics, marks the onset of incommensurate finite-range spin-spin correlations in the chain (see, e.g., Ref. [2] and references therein). In the region $J>J_{D}$ the poles in Eq. (9) move away from the imaginary axis. Due to the emergence of a real part, incommensurate real-space spin-spin correlations appear. Later on, using also precise DMRG data, we shall discuss in more detail the physics of the disorder point $J_{D}$.

\section{DMRG AND EXACT-DIAGONALIZATION ANALYSIS}

To obtain quantitative results, we have used DMRG and Lanczos exact-diagonalization methods. DMRG is particularly adapted to the problem, for it allows treating large enough frustrated systems. DMRG is also free from the negative sign problems characteristic of 
Monte Carlo methods when applied to frustrated systems. We have studied three types of open spin chains, $\left(S_{1}, S_{2}\right)=(3 / 2,1),(1,1 / 2)$, and $(3 / 2,1 / 2)$, containing up to $N=20$ unit cells, many times the observed correlation lengths. The DMRG results for the ground-state energy and sublattice magnetization are presented in Table 1. The sublattice magnetization, measuring the long-range antiferromagnetic order in the ferrimagnetic state, displays only a slight monotonic decrease and remains finite up to the transition point $J_{T}$ discussed below.

An analysis of the correlations calculated by DMRG for large values of frustration is difficult due to incommensurability. As to the small frustration region, the behavior of the transverse spin-spin correlations can clearly be extracted for distances $r$ up to 20 ( the limit is due to the very small correlation length $\xi$, leading to a very fast decay to numerical noise). Fits clearly reveal that the planar correlations obey the $2 D$ Ornstein-Zernike form, Eq. (11), in accord to the qualitative LSWT analysis. The fit can be done over many orders of magnitude. A fit to a purely exponential law is much worse. The correlation lengths given in Table 2 are extracted from these fits. As an example, in Fig. 6 we show the correlation length $\xi$ vs. $J$ in the case $(1,1 / 2)$. We recognize a typical behavior for the function $\xi(J)$, showing the existence of a disorder point at $J \approx 0.177$. Recently, it has been argued by one of the authors [2], that in quantum spin chains at $T=0$ the presence of a phase transition from a commensurate to an incommensurate phase in the classical limit should be generically reflected in the presence of so-called disorder points, a concept of classical statistical mechanics. Indeed, such disorder points have already been identified in a variety of cases, such as frustrated spin chains, as well as in the AKLT model [2]. We have clearly found such quantum remnants of the classical phase transition also in the present model, Table 3. The point $J_{D}$ is characterized by the onset of incommensurate real-space correlations as well as by an infinite derivative of the correlation length $d \xi / d J$ at $J=J_{D}-0$. We were not able to extract conclusively at the disorder point the crossover from two-dimensional to one-dimensional Ornstein-Zernike correlation functions, due to the extreme shortness of the correlation length. Generically, it can be expected that the disorder point shifts to smaller values of frustration for larger $w$, as it has been borne out by our 
numerical results as well as by the spin-wave analysis, Eq. (10).

Not as satisfying data could be obtained for the $\left\langle S^{z} S^{z}\right\rangle$-correlations: As we are working in a fixed $S_{\text {total }}^{z} \neq 0$ environment, we have to subtract one-site expectation values $\left\langle S^{z}\right\rangle$. These values introduce substantial errors, given that the actual correlation is smaller by orders of magnitude than the subtracted value; in addition, the longitudinal correlation length is shorter than the transverse one.

Finally, let us consider the structure of the low-energy levels around the classical transition as obtained from the DMRG and ED simulations. Both methods seem to be effective tools revealing a complicated picture of low-energy level crossings. At the transition point $J_{T}>J_{C}$ (see Table 3) a first-order transition (level crossing) takes place from the ferrimagnetic state with $S_{g}=\left(S_{1}-S_{2}\right) N$ to a singlet $S_{g}=0$ state. This is accompanied by a basic rearrangement of the spin correlations. Beyond the transition point $J_{T}$, the ED numerical analysis indicates additional level crossings. To be specific, we illustrate that for a periodic system with $N=6$ cells and $\left(S_{1}, S_{2}\right)=(1,1 / 2)$ : The discontinuous transition from the ferrimagnetic ground state with total spin $S_{g}=\left(S_{1}-S_{2}\right) N=3$ to the ground state with $S_{g}=0$ appears at $J=0.229$. This singlet ground state is stable up to $J=0.260$. In the next interval $(0.260,0.303)$ the lowest energy level is a triplet, $S_{g}=1$. Beyond the point $J \approx 0.303$ the ground state has $S_{g}=0$, but there are several crossings between singlets of different symmetries at $J \approx 0.417,0.735$, and 0.759 . These may however be due to incommensurability effects of short chains. DMRG shows that at least from $J=0.25$ onwards the ground state has $S_{g}=0$; for values between the transition and $J=0.24$, numerically a $S_{g}=1$ ground state cannot be clearly excluded due to a very small spacing between the levels just above the transition and at the same time a large DMRG error close to the transition. Definitely, no higher $S_{g}$ is observed for the ground state. Together with the ED result, we believe however that the ground state is indeed $S_{g}=0$. 


\section{DISCUSSION}

In this paper the emphasis was on relatively small values of the frustration parameter $J$, when the classical ferrimagnetic state survives the zero-point spin fluctuations. As a whole, frustration produces a strong reduction of the finite-range spin-spin correlation length. Our key results are: (i) The quantum spin fluctuations destroy the classical continuous transition and generate a discontinuous transition from the long-range ordered ferrimagnetic $S_{g}=\left(S_{1}-S_{2}\right) N$ state to a singlet state, $S_{g}=0$. The transition point $J_{T}$ lies beyond the classical transition point $J_{C}$. (ii) The discontinuous transition point $J_{T}$ is preceded by a disorder point $J_{D}<J_{T}$, marking the onset of incommensurate real-space short-range correlations. For smaller values of frustration, $J<J_{D}$, the transverse short-range spin-spin correlations show typical $2 D$ Ornstein-Zernike behavior.

Even in the small $J$ region, a number of open issues can be indicated. One of the important questions concerns the nature of the $S_{g}=0$ ground states established beyond the ferrimagnetic phase. As mentioned above, the classically broken $\mathrm{SO}(3)$ symmetry in the spiral state is generally expected to be restored by quantum fluctuations in the onedimensional systems. Thus, we are enforced to look for possible magnetically disordered states. A valuable information can be obtained from the Lieb-Schultz-Mattis theorem [16] adapted to mixed chain spin models [6]. In our case, the theorem is applicable to the systems $\left(S_{1}, S_{2}\right)=(1,1 / 2)$ and $(3 / 2,1)$, and says that the model (1) either has gapless excitations or else has degenerate ground states. Thus, we should look for phases with presumably some discrete symmetry broken. An analysis of possible quantum spin phases has already been implemented in the context of the model of frustrated ferromagnetic chains $\left(J_{1}<0\right.$,

$\left.J_{2}>0\right)$ 17, 18. In particular, two possible nematiclike phases with additional symmetry breaking of reflections about a bond or about a site have been suggested [17]. It was argued above that the antiferromagnetic excitations in our model do not play any important role near $J_{T}$ due to the large energy gap $\Delta$ near the transition. Therefore, the instability of the ferrimagnetic state is expected to capture the main features of the ferromagnetic instability 
appearing in the frustrated ferromagnetic chain. However, in contrast to the cascade of transitions predicted for the ferromagnetic chain [18], our numerical results point towards a direct transition from the ferrimagnetic $S_{g}=\left(S_{1}-S_{2}\right) N$ state to a state with $S_{g}=0$. Such a difference in the behavior of both models at $J_{T}$ may be attributed to the low-energy multimagnon bound states appearing in the ferromagnetic chain. In addition, the variety of possible nonmagnetic quantum phases beyond $J_{T}$ may be considerably enlarged due to the existence of two kinds of site spins, $S_{1}$ and $S_{2}$, in the mixed chain model. The complicated picture of low-energy level crossings described above points towards a rich phase diagram of the discussed ferrimagnetic model, as well. Studies in this direction are in progress at the moment.

\section{ACKNOWLEDGMENTS}

One of the authors (N.B.I.) thanks the staff of the Institut für Theoretische Physik, Magdeburg, for hospitality. The stay in the Universität Magdeburg was supported by

the Deutsche Forschungsgemeinschaft (436 BUL 17/9/97). J.R. thanks the Deutsche Forschungsgemeinschaft for support (project Ri 615/1-2). 


\section{REFERENCES}

[1] R. Bursill, G. A. Gehring, D. J. J. Farnell, J. B. Parkinson, T. Xiang, and C. Zeng, J. Phys.: Condens. Matter 7, 8605 (1995); R. Citra, S. Pati, H. R. Krishnamurthy, D. Sen, and S. Ramasesha, Phys. Rev. B 52, 6581 (1995).

[2] U. Schollwöck, Th. Jolicoeur, and T. Garel, Phys. Rev. B 53, 3304 (1996); A. Kolezhuk, R. Roth, and U. Schollwöck, Phys. Rev. Lett. 77, 5142 (1996); Phys. Rev. B 55, 8928 (1997).

[3] S. R. White and I. Affleck, Phys. Rev. B 54, 9862 (1996).

[4] S. K. Pati, S. Ramasesha, and D. Sen, Phys. Rev. B 55, 8894 (1997); J. Phys.: Condens. Matter 9, 8707 (1997); A. K. Kolezhuk, H.-J. Mikeska, and S. Yamamoto, Phys. Rev. B 55, R3336 (1997); S. Brehmer, H.-J. Mikeska, and S. Yamamoto, J. Phys.: Condens. Matter 9, 3921 (1997); T. Ono, T. Nishimura, M. Katsumura, T. Morita, and M. Sugimoto, J. Phys. Soc. Jpn. 66, 2576 (1997).

[5] G.-S. Tian, Phys. Rev. B 56, 5355 (1997).

[6] T. Fukui and N. Kawakami, Phys. Rev. B 55, R14709 (1997); ibid. 56, 8799 (1997).

[7] Y. Xian, Phys. Rev. B 52, 12485 (1995); H. Niggemann, G. Uimin, and J. Zittartz, J. Phys.: Condens. Matter 9, 9031 (1997); N. B. Ivanov and J. Richter, Phys. Lett. A 232, 308 (1997).

[8] O. Kahn, Magnetism of the Heteropolymetallic Systems, Structures and Bonding (Springer -Verlag, Berlin, 1987), Vol. 68, p. 91; G. T. Yee, J. M. Manriquez, D. A. Dixon, R. S. McLean, D. M. Groski, R. B. Fippen, K. S. Narayan, A. J. Epstein, and J. S. Miller, Adv. Mater. 3, 309 (1991).

[9] H. J. de Vega and F. Woynarovich, J. Phys. A 25, 4499 (1992); S. R. Adams and M. J. Martins, ibid. 26, L529 (1993); F. C. Alcaraz and A.L. Malvezzi, ibid. 30, 767 (1997); M. Fujii, S. Fujimoto, and N. Kawakami, J. Phys. Soc. Jpn. 65, 2381 (1996). 
[10] N. B. Ivanov, preprint, cond-mat/9712014.

[11] P. Chandra and B. Doucot, Phys. Rev. B 38, 9335 (1988).

[12] E. Lieb, and D. Mattis, J. Math. Phys. (N.Y.) 3, 749 (1962).

[13] J. Richter, N. B. Ivanov, and K. Retzlaff, Europhys. Lett. 25, 545 (1994).

[14] P. Azaria, B. Delamotte, and T. Jolicoeur, Phys. Rev. Lett. 64, 3175 (1990).

[15] J. Igarashi, J. Phys. Soc. Jpn. 62, 4449 (1993).

[16] E. H. Lieb, T. Schultz, and D. J. Mattis, Ann. Phys. (N.Y.) 16, 407 (1961).

[17] A. V. Chubukov, Phys. Rev. B 44, 4693 (1991).

[18] V. Ya. Krivnov and A. A. Ovchinnikov, Phys. Rev. B 53, 6435 (1996). 


\section{FIGURES}

FIG. 1. The mixed-chain Heisenberg spin model studied in the paper. $J_{1}, J_{2}>0$.

FIG. 2. The dispersion functions $E_{k}^{(a, b)}$ in a first-order spin-wave approximation for different values of the frustration parameter $J$.

FIG. 3. The antiferromagnetic gap $\Delta$ vs. $J$ obtained in a first-order spin-wave approximation (solid curves) and by the Lanczos exact-diagonalization method.

FIG. 4. The ground-state energy per cell $E_{0}$ of the frustrated $(1,1 / 2)$ ferrimagnetic chain obtained by the second-order SWT, DMRG, and the exact-diagonalization methods.

FIG. 5. On-site magnetization of the first sublattice $m_{1}$ in the frustrated $(1,1 / 2)$ ferrimagnetic chain obtained by LSWT, DMRG, and the exact-diagonalization methods. The second-order spin-wave results are not conclusive, as explained in the text.

FIG. 6. The transverse spin-spin correlation length in the frustrated $(1,1 / 2)$ system vs. $J$ obtained from the LSWT and the DMRG method. $J_{D} \approx 0.177$ is the disorder point marking the onset of incommensurate real-space finite-range spin-spin correlations. 


\section{TABLES}

TABLE I. DMRG results for the ground-state energy per cell $E_{0}$, and the on-site sublattice

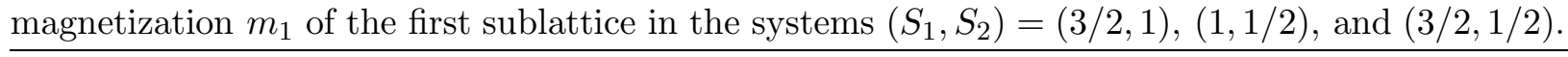

\begin{tabular}{|c|c|c|c|c|c|c|}
\hline \multirow[b]{2}{*}{$J$} & \multicolumn{2}{|c|}{$\left(\frac{3}{2}, 1\right)$} & \multicolumn{2}{|c|}{$\left(1, \frac{1}{2}\right)$} & \multicolumn{2}{|c|}{$\left(\frac{3}{2}, \frac{1}{2}\right)$} \\
\hline & $E_{0}$ & $m_{1}$ & $E_{0}$ & $m_{1}$ & $E_{0}$ & $m_{1}$ \\
\hline 0.00 & -1.93096 & 1.14428 & -1.45409 & 0.79249 & -1.96723 & 1.35743 \\
\hline 0.02 & -1.90345 & 1.13828 & -1.43337 & 0.78995 & -1.92173 & 1.35336 \\
\hline 0.04 & -1.87614 & 1.13198 & -1.41278 & 0.78730 & -1.87639 & 1.35319 \\
\hline 0.06 & -1.84906 & 1.12535 & -1.39234 & 0.78455 & -1.83121 & 1.35092 \\
\hline 0.08 & -1.82221 & 1.11838 & -1.37206 & 0.78167 & -1.78621 & 1.34853 \\
\hline 0.10 & -1.79561 & 1.11099 & -1.35194 & 0.77866 & -1.74140 & 1.34604 \\
\hline 0.12 & -1.76928 & 1.10319 & -1.33199 & 0.77551 & -1.69679 & 1.34339 \\
\hline 0.14 & -1.74325 & 1.09489 & -1.31223 & 0.77272 & -1.65239 & 1.34061 \\
\hline 0.16 & -1.71755 & 1.08608 & -1.29267 & 0.76871 & -1.60822 & 1.33767 \\
\hline 0.18 & -1.69219 & 1.07668 & -1.27332 & 0.76504 & -1.58472 & \\
\hline 0.20 & -1.66722 & 1.06662 & -1.25420 & 0.76115 & & \\
\hline 0.22 & -1.64267 & 1.05582 & -1.23533 & 0.75702 & & \\
\hline 0.24 & -1.61859 & 1.04418 & -1.220 & & & \\
\hline 0.26 & -1.59502 & 1.03157 & -1.214 & & & \\
\hline 0.28 & -1.5720 & 1.01785 & -1.212 & & & \\
\hline
\end{tabular}


TABLE II. DMRG results for the transverse spin-spin correlation length $\xi$ in the systems $\left(S_{1}, S_{2}\right)=(3 / 2,1),(1,1 / 2)$, and $(3 / 2,1 / 2)$ for different values of the frustration parameter $J$.

\begin{tabular}{cccccccccccc}
\hline \hline $\mathrm{J}$ & 0.00 & 0.02 & 0.04 & 0.06 & 0.08 & 0.10 & 0.12 & 0.14 & 0.16 & 0.18 & 0.20 \\
\hline$(3 / 2,1)$ & 1.74 & 1.67 & 1.60 & 1.53 & 1.45 & 1.37 & 1.29 & 1.20 & 1.11 & 1.01 & 0.89 \\
$(1,1 / 2)$ & 1.01 & 0.97 & 0.93 & 0.89 & 0.84 & 0.80 & 0.74 & 0.69 & 0.61 & 0.46 & 0.50 \\
$(3 / 2,1 / 2)$ & 0.75 & 0.72 & 0.67 & 0.64 & 0.56 & 0.53 & 0.45 & & & & \\
\hline \hline
\end{tabular}

TABLE III. DMRG results for the disorder point $J_{D}$ and the first-order transition point $J_{T}$ found by DMRG in the systems $\left(S_{1}, S_{2}\right)=(3 / 2,1),(1,1 / 2)$, and $(3 / 2,1 / 2) . \quad J_{C}=w / 2\left(w^{2}+1\right)$ is classical commensurate-incommensurate transition point. $J_{D}^{s w}=1 / 2(w+1)$ is the LSWT result for the disorder point.

\begin{tabular}{lcccc}
\hline \hline$\left(S_{1}, S_{2}\right)$ & $J_{D}^{s w}$ & $J_{D}(D M R G)$ & $J_{C}$ & $J_{T}(D M R G)$ \\
\hline$(3 / 2,1)$ & $1 / 5$ & $0.235(5)$ & $3 / 13$ & $0.280(1)$ \\
$(1,1 / 2)$ & $1 / 6$ & $0.177(2)$ & $1 / 5$ & $0.231(1)$ \\
$(3 / 2,1 / 2)$ & $1 / 8$ & $0.14(2)$ & $3 / 20$ & $0.161(1)$ \\
\hline \hline
\end{tabular}




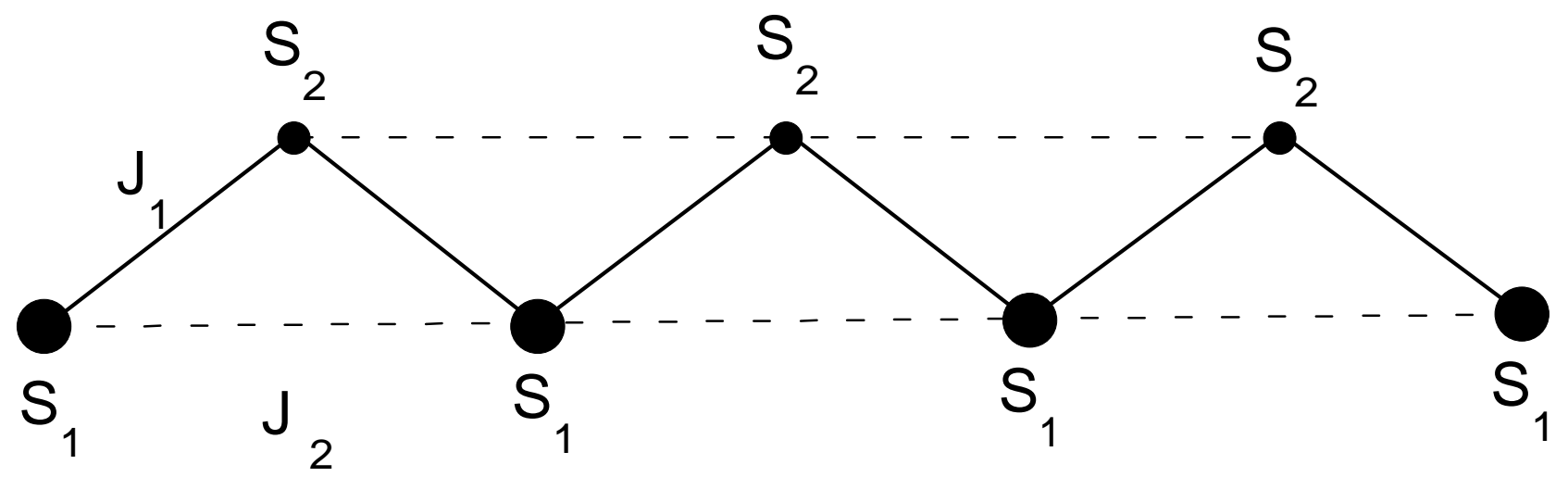

Fig. 1: N. B. Ivanov et al. 
Fig. 2: N. B. Ivanov et al.

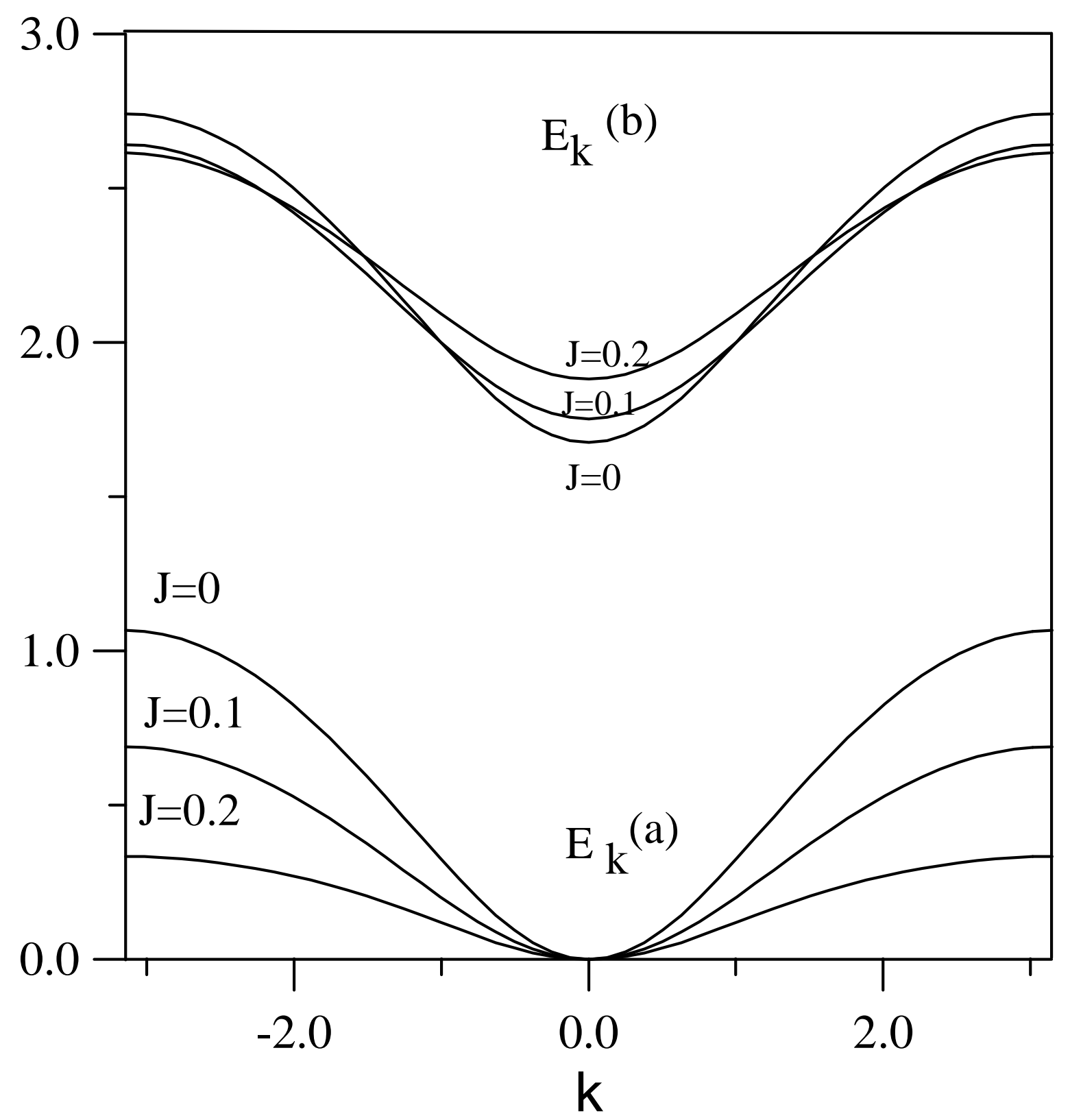


Fig. 3: N. B. Ivanov et al.

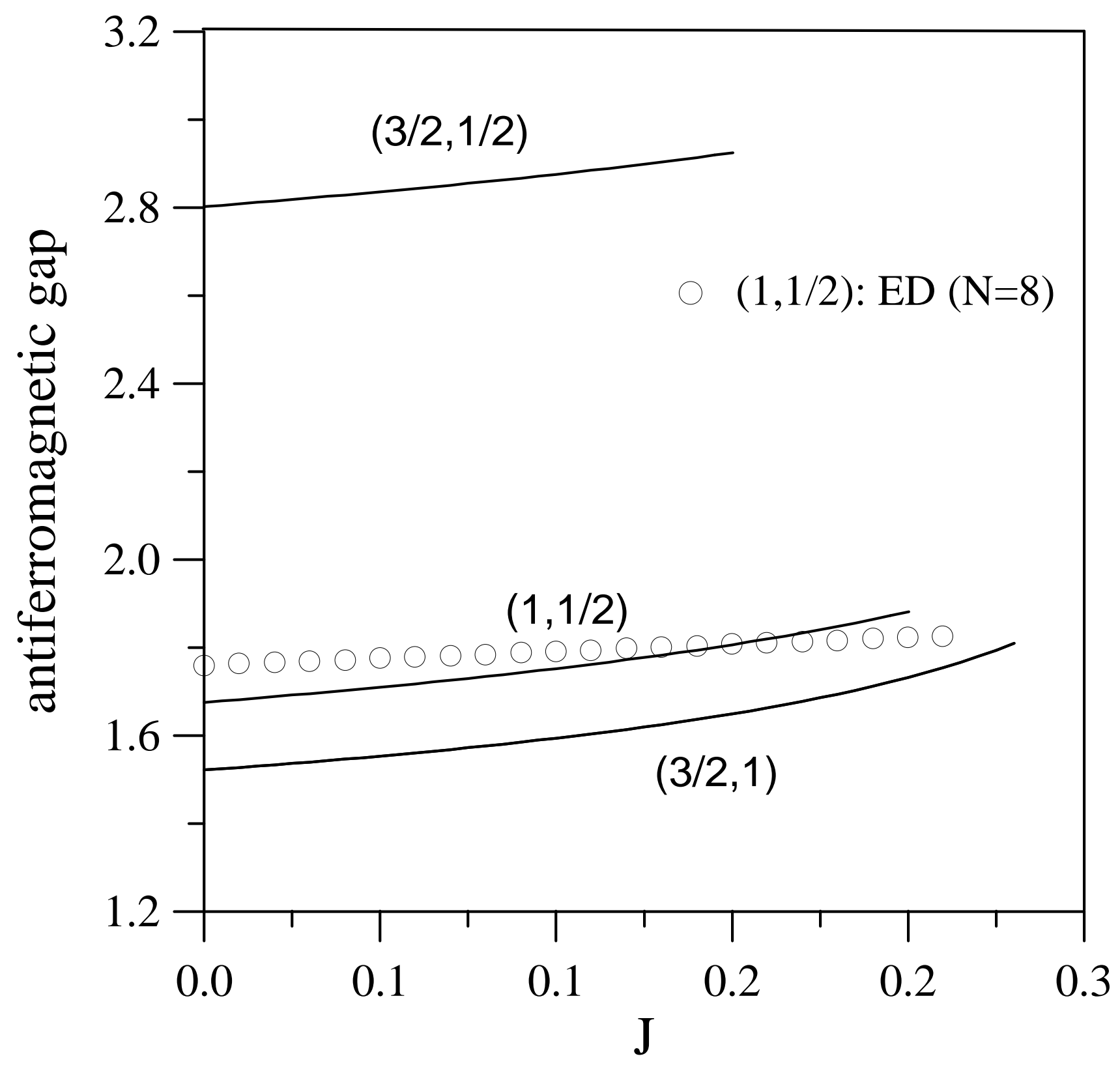


Fig. 4: N. B. Ivanov et al.

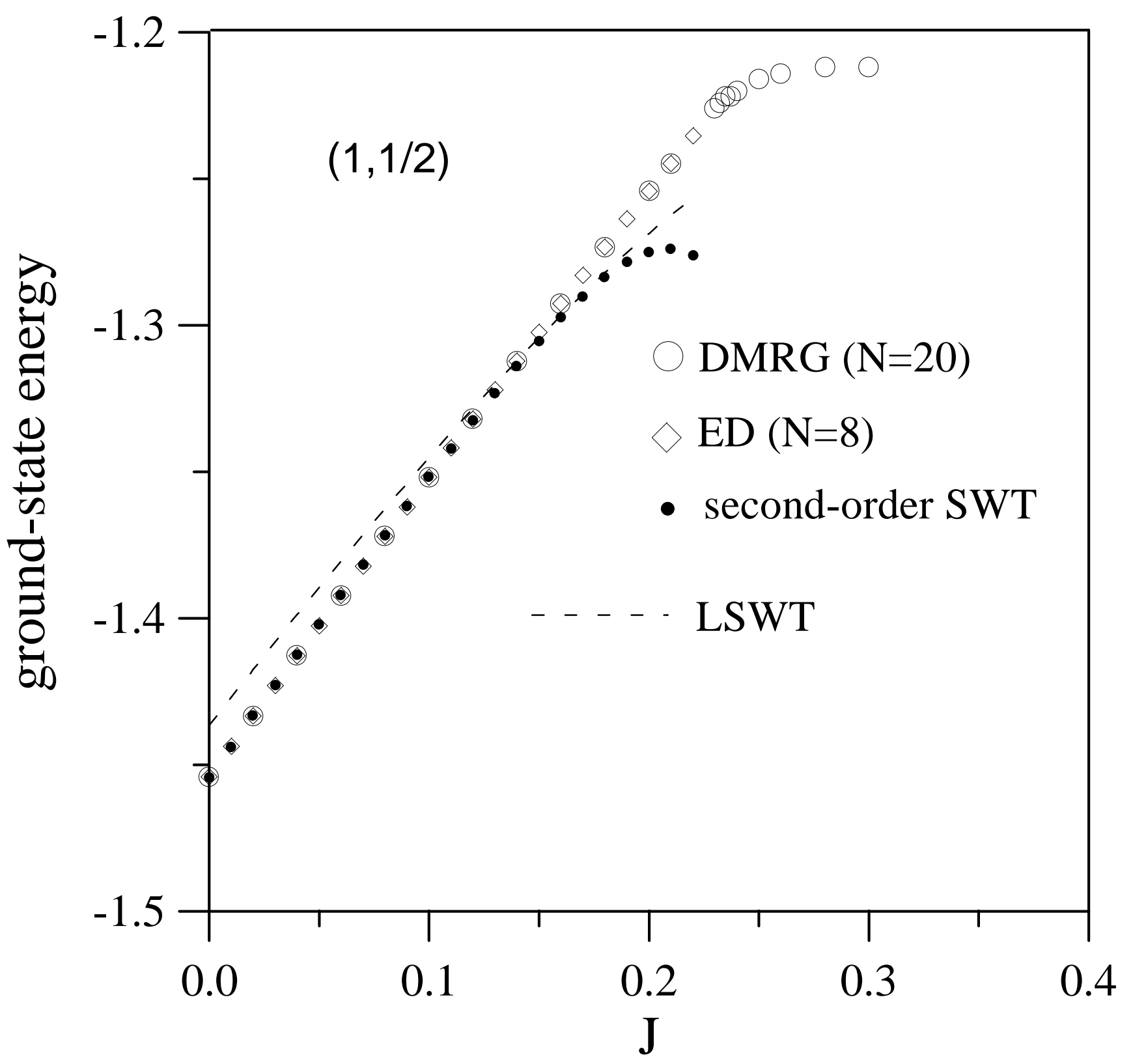


Fig. 5: N. B. Ivanov et al.

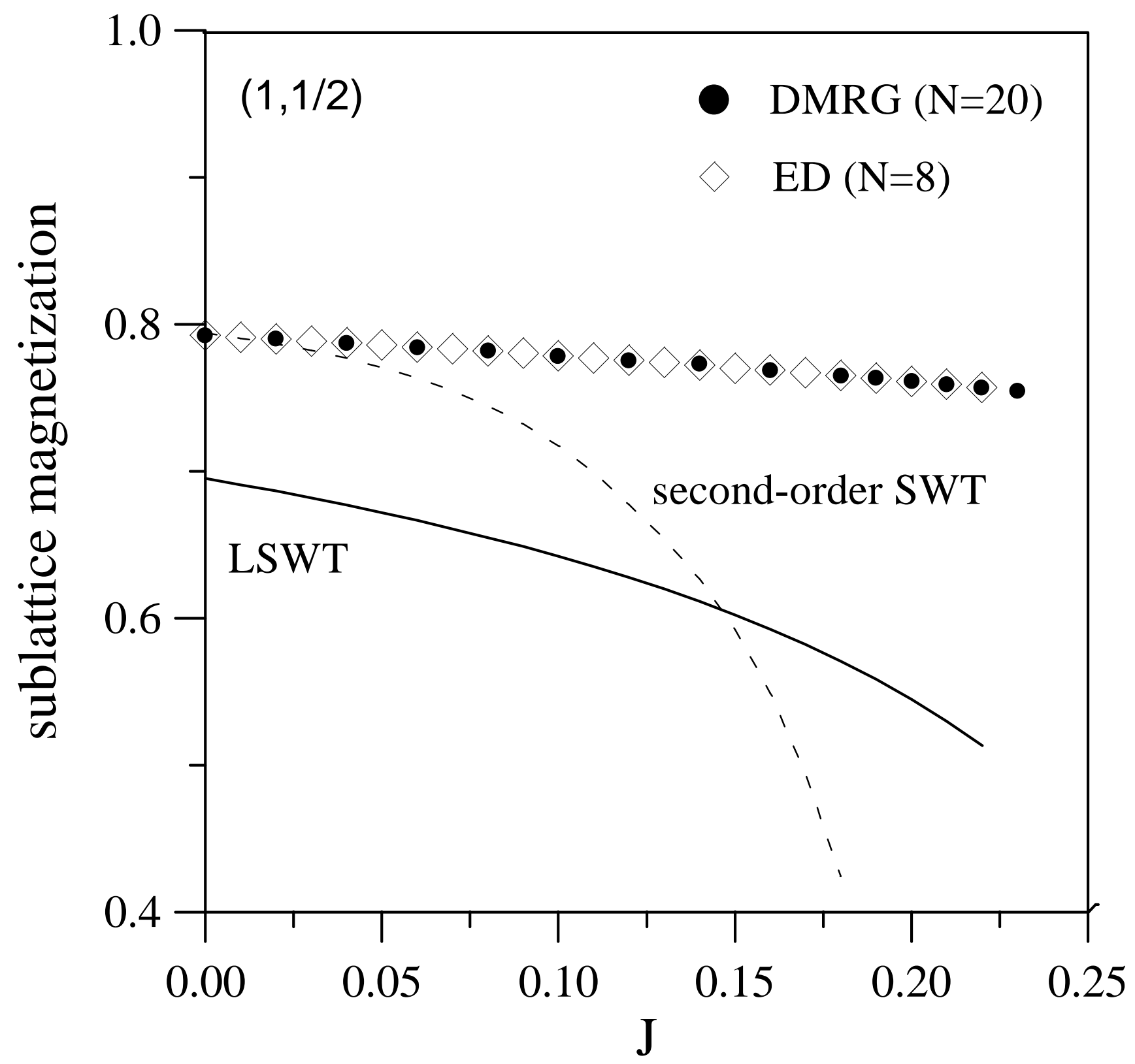


Fig. 6: N. B. Ivanov et al.

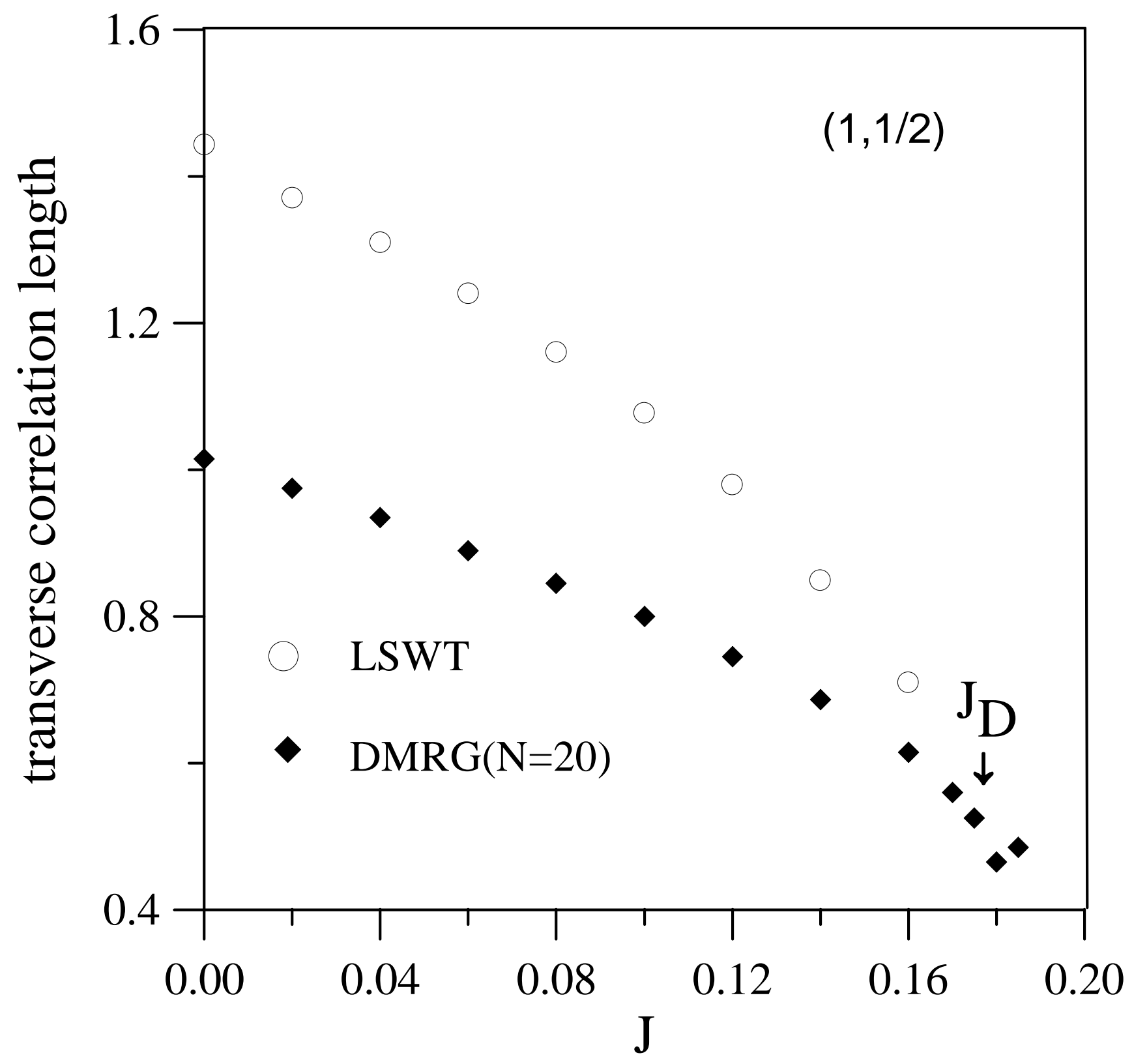

